Perspective

\title{
Good practice guide for papers on batteries for the Journal of Power Sources
}

\author{
Jie Li $^{\text {a, *, Catia Arbizzani }}{ }^{\mathrm{b}}$, Signe Kjelstrup ${ }^{\mathrm{c}}$, Jie Xiao ${ }^{\mathrm{d}}$, Yong-yao Xia ${ }^{\mathrm{e}}$, Yan Yu ${ }^{\mathrm{f}}$, Yong Yang ${ }^{\mathrm{g}}$, \\ Ilias Belharouak ${ }^{\mathrm{h}}$, Thomas Zawodzinski ${ }^{\mathrm{i}}$, Seung-Taek Myung ${ }^{\mathrm{j}}$, Rinaldo Raccichini ${ }^{\mathrm{k}}$, \\ Stefano Passerini ${ }^{1, \mathrm{~m}}$ \\ ${ }^{a}$ Helmholtz-Institute Münster (IEK-12), Forschungszentrum Jülich GmbH, Corrensstr. 46, Münster, 48149 Germany \\ b Department of Chemistry "Giacomo Ciamician", Alma Mater Studiorum Università di Bologna, Via F. Selmi 2, Bologna, 40126 Italy \\ ${ }^{\mathrm{c}}$ PoreLab, Department of Chemistry, Norwegian University of Science and Technology, NO-7491 Trondheim, Norway \\ ${ }^{d}$ Energy and Environmental Directorate, Pacific Northwest National Laboratory, Richland, WA, 99352 United States \\ ${ }^{\mathrm{e}}$ Department of Chemistry, Institute of New Energy, Fudan University, Shanghai, 200433 China \\ ${ }^{\mathrm{f}}$ Department of Material Science \& Engineering, University of Science and Technology of China (USTC), Hefei, 23052 China \\ ${ }^{g}$ State Key Laboratory for Physical Chemistry of Solid Surfaces, Department of Chemistry, Xiamen University, Xiamen, 361005 China \\ ${ }^{\mathrm{h}}$ Energy and Transportation Science Division, Oak Ridge National Laboratory, Oak Ridge, TN 37830, United States \\ ${ }^{\mathrm{i}}$ University of Tennessee Knoxville, Knoxville, TN, United States \\ ${ }^{\mathrm{j}}$ Faculty of Nanotechnology and Advanced Materials Engineering, Sejong University, Seoul, 143-747 South Korea \\ ${ }^{\mathrm{k}}$ National Physical Laboratory, Hampton Road, Teddington, Middlesex, TW11 OLW, United Kingdom \\ ${ }^{1}$ Electrochemistry I, Helmholtz Institute Ulm, Helmholtzstr. 11, Ulm, 89081 Germany \\ ${ }^{\mathrm{m}}$ Karlsruhe Institute of Technology, P.O. Box 3640, Karlsruhe, 76021, Germany
}

\section{Good Practice for the introduction section}

In the introduction (i.e., the initial section of a scientific paper) it is mandatory to present the topic of the research and state the background and relative rationale (if applied). In this section the authors should also explain the aim and novelty of the research work. It is not necessary, except for review papers, to describe the full history of well-known types of batteries (especially for lithium ion batteries and sodium ion batteries, for which many reviews are available in literature), but instead to emphasize the state-of-the-art and challenges of the specific research topic. The cited literature should be relevant and up-to-date, i.e., results from references published more than five years ago (three years for hot topics, such as Ni-rich cathodes for lithium ion batteries or solid-state electrolytes) cannot be considered up-to-date. No detailed conclusionstyle paragraph should be included in the introduction section.

\section{Good Practice for the experimental section}

Depending on the topic, the experimental section should include synthesis procedures, physicochemical characterization experiments, cell/battery assembly and electrochemical testing. The following information must be considered for the experimental section:

1. Synthesis procedures: The method(s) should be described in an accurate and precise way and, if appropriate, relevant references can be used. The scale of synthesis (e.g., in the gram or kilogram range), the supplier and the purity of starting reagents, and reaction conditions (e.g., concentration, $\mathrm{pH}$, temperature etc.) must be disclosed where possible. In the case of "fast" synthetic procedures, such as "one-step" reactions, only direct synthetic processes can be considered; that is, methods of synthesis including precursor preparation and post-calcination steps cannot be considered as "one-step" reactions.

2. Physicochemical characterization experiments: Routine characterization techniques such as X-ray diffraction (XRD), Scanning Electron Microscopy (SEM) or Raman etc. spectroscopy can be described in terms of technical specification of the experimental setup used [1]. Custom-built setups for specific measurements are welcomed but they must be precisely described and illustrated with a schematic [2]. If a specialist method or technique is applied, a brief introduction to the scientific background and corresponding program of data processing is suggested [3]. For instance, for X-ray absorption spectroscopy (XAS) measurements, the sample preparation, beamline location, type of monochromator and the energy and mode of XAS measurements [4] must be disclosed for the benefit of the reader.

3. Cell/battery assembly: It is important to introduce and report the following specifications: (i) cell type and configuration (e.g., glass cell, coin cell, pouch cell, three-electrode cell etc.), (ii) mass composition of the electrode (i.e., active material, binder and

\footnotetext{
* Corresponding author.

E-mail address: jie.li@fz-juelich.de (J. Li).
} 
electronically conductive additive), (iii) type and number of separator layers used, (iv) electrolyte formulation and volume, as well as water content in the case of non-aqueous electrolytes and (v) environment of the cell assembly (e.g., dry room or glovebox) with relevant specifications (e.g., dewpoint, $\mathrm{H}_{2} \mathrm{O}$ and $\mathrm{O}_{2}$ levels). When describing the electrode preparation, the electrode area and the mass loading of active material must be given [5]. If the research focus is high-power application, the total amount of carbon in the electrode should be reported to facilitate meaningful comparison with data available in the literature. In the case of multiple cell testing, the amount of electrolyte used in each cell should also be reported for impartial comparison.

4. Electrochemical testing: With the exception of testing conditions provided by the manufacturers of commercial devices, for lab-scale electrochemical experiments the rationale for the testing conditions and the aim of each electrochemical experiment should be clearly explained. In general, besides reporting the environmental temperature of testing, the potential range and the sweep rate are required for voltammetry measurements [6]. The frequency range, the magnitude of the perturbation (either current or potential) and the rest time applied are required for electrochemical impedance spectroscopy (EIS) measurements. When EIS measurements are presented in a complex impedance plot (generally called a Nyquist plot), this must be squared and ortho-normed (i.e., same scale on $\mathrm{X}$ and $\mathrm{Y}$ axes), with raw impedance data reported as symbols and fitted data as lines [7]. If equivalent circuit elements are used to fit the EIS data to obtain quantitative derived parameters, the equivalent circuit and the error between the raw and fitted data must be reported. Concerning galvanostatic cycling, in the case of three-electrode cell set-up (either half-cell or full cell testing), the chemistry and the geometry of the reference electrode must be specified [8]. In the case of two-electrode cell testing (i.e., full cell experiments) the cell voltage must be reported. More generally, the voltage range and the theoretical capacity (if available) must be disclosed.

\section{Good Practice for the results and discussion section}

Research works in the field of batteries must demonstrate the capability of the system under investigation to store energy by means of efficient reversible electrochemical reactions. In this regard, the following specifications must be provided when a manuscript is submitted to the Journal of Power Sources.

1. The specific capacity (defined as the total amount of charge that can be stored per unit volume or mass) should always be reported together with the applied current. In a full cell test, the value of the capacity should be reported (without ambiguity) with respect to either the mass of the cathode, the mass of the anode, or the total mass of active material. To evaluate cell balancing, the mass loading ratio between negative and positive electrodes [9] is also required when reporting full cell data. If any pre-activation strategy is applied before full cell assembly [10], it must be clearly reported. If no pre-activation strategy is used, the charge/discharge coulombic efficiency of the initial cycle must never be omitted.

2. When cycling performance is reported, the results should be presented together with the testing temperature, voltage range and applied current. The corresponding coulombic efficiency value should also be disclosed. The minimum number of cycles required to demonstrate sufficiently credible electrochemical energy storage capability depends on the type of material(s) under investigation and on the cell configuration used.

a. In the case of well-established active materials for lithium/ sodium ion batteries, at least 100 cycles are expected. Low specific currents ( $\leq 50 \mathrm{~mA} \mathrm{~g}^{-1}$ ) should be used for cycling stability tests. In the case of rate capability tests, specific currents in the range 25 $\mathrm{mA} \mathrm{g}^{-1}$ to $250 \mathrm{~mA} \mathrm{~g}^{-1}$ are required. b. When reporting "new" active materials (e.g., as a new structure or new formula), authors must focus, besides the disclosure of the synthetic procedure, on the electrochemical behavior in battery configuration in order to be considered by the Journal of Power Sources. Thus, at least a cyclic voltammetry experiment or a voltage profile associated with the first cycle of metal-ion intake/ release is required, supported by detailed explanation of active material reactions [11].

3. A demonstration of the electrochemical stability window is required for any submission on electrolytes (linear sweep voltammetry and/or relevant cycling data must be reported and discussed) [6]. Electrode and/or cell self-discharge measurements are also recommended (preferably at full state of charge). This aspect is particularly relevant for highly concentrated electrolytes (e.g., water-in-salt electrolytes), and for high-voltage and organic/polymeric electrode materials. In the case of solid-state electrolytes, the temperature-dependent conductivity should be also measured and discussed in the manuscript.

4. In the case of cyclic voltammetry experiments for the evaluation of the diffusion coefficient, if the peak potential varies with scan rate the system should be treated as electrochemically irreversible and therefore the Randles-Ševčík equation cannot be applied. In this regard, it is suggested to verify the linearity of the peak current vs. square root of scan rate [12].

5. When a new active material is reported to surpass the state-of-theart in terms of performance, a comparison (either in tabular or in graphical form) with the data from the existing literature must be provided [13]. When discussing the comparison, the authors must specify if the data were acquired under similar testing conditions. Especially for rate capability tests, the mass loading, the applied current and the voltage range are critical in terms of electrochemical performance. Particularly, the specific current (expressed, e.g., as $\mathrm{mA}$ $\mathrm{g}^{-1}$ or $\mathrm{A} \mathrm{g}^{-1}$ ) and current density (expressed, e.g., as $\mathrm{mA} \mathrm{cm}{ }^{-2}$ or $\mathrm{mA}$ $\mathrm{cm}^{-3}$ ) must be clearly reported.

When reporting new ceramic compounds as active materials or as solid-state electrolytes for batteries, the chemical composition must be confirmed by elemental analysis methods (e.g., inductively coupled plasma mass spectrometry (ICP-MS)). Energy-dispersive X-ray (EDX) surface mapping, obtained during SEM measurement, can be considered as supporting analysis, but not as clear evidence of bulk chemical composition. It is also recommended to characterize the structure and the purity of the compounds by structural analysis (e.g., XRD or neutron diffraction). The software/program used, the structural model applied, the correlation coefficient ( $\mathrm{R}$ factor) and the uncertainty of each value must be clearly reported when refinement is applied.

A large number of manuscripts investigate modification (e.g., bulk substitution or surface modification) of conventional active materials through a variety of doping and coating procedures (with specific elements and/or compounds). The rationale for improvement is often a merely "copy/paste" exercise from previously published research works without any support from experimental results. Similar issues can be found for submissions related to materials with "enhanced" morphology. Indeed, a vast number of papers have been published in recent years, mainly focusing on the size or shape aspects of the material under study. In this regard, the Journal of Power Sources is no longer interested in manuscripts where the main discovery or innovation is the shape or size of a material, unless significant advantages in term of performance, cost or sustainability are clearly demonstrated. In particular, authors should avoid inventing novel names for materials, and should ensure that micrographs are representative and that robust statistical information is presented.

In the case of studies dealing with electrolytes containing new component(s), (e.g., additives, solvents or salts), it is necessary to carry out measurements with an adequately selected benchmark system [14]. For example, if a submission reports research work on an additive for 
propylene carbonate-based electrolyte, the direct comparison of experimental results with the data obtained from a conventional carbonate-based electrolyte cannot be considered as scientifically appropriate. The electrolyte compatibility with active materials and binders (for lithium and sodium ion batteries) is another important aspect which should be considered. Electrochemical testing of the electrolyte in full cell configuration containing conventional binders, cathodes and anodes is highly encouraged [15]. Measurements of mechanical properties (e.g., tensile testing and/or Young's modulus) are suggested for research works on polymer electrolytes or hybrid electrolytes with polymer components [16]. In the case of solid-state electrolytes, the sample preparation for conductivity measurement and the battery/cell assembly procedure should be described in detail [17].

When results from cell disassembly and post-mortem analysis are presented, it is necessary to describe the sample preparation in detail. The procedure for cleaning/rinsing the electrode after disassembly of a cycled cell/battery has a significant influence on the analysis outcome and, thus, must be clearly reported. Details regarding the conditions for post-mortem electrode harvesting (such as the type of solvent used for the rinsing or dipping procedure) and subsequent electrode drying should be defined. For materials associated with severe self-charge/selfdischarge issues, the post-mortem experiment needs to be designed to avoid any significant change in the state of charge/discharge in the time between disconnection of the cell from the battery tester and the disassembly of the cell under inert conditions.

In a substantial number of submissions, the terms "in situ" and "operando" have been applied incorrectly. In the case of cell testing, the term "in situ" should be applied for measurements probing specific changes (e.g., structural or chemical) under relevant operation conditions (e.g., by interrupting electrochemical experiments at specific state of charge/discharge of the cell). The term "operando" combines in situ investigation (e.g., spectroscopic) with simultaneous real-time monitoring of the electrochemical performance of the cell (no interruption of cell charge/discharge is required). Examples of meaningful use of these terms could be "high temperature in situ XRD measurements" or "operando differential electrochemical mass spectroscopy". Operando measurements are generally carried out using a complex device or specific cell set-up [18-21], which should be reported and described in the manuscript.

For papers concerning metal batteries (in which the negative electrode is metallic, i.e. $\mathrm{Li}, \mathrm{Na}, \mathrm{K}, \mathrm{Mg}, \mathrm{Ca}, \mathrm{Al}, \mathrm{Zn}$, etc.), the excess of the active metal on the negative electrode must be taken into account when reporting and interpreting the calculated coulombic efficiency. This applies equally to initial excess due to the use of a pure metal foil as the negative electrode, and to accumulated excess due to negative electrodes in which the active metal is plated onto a substrate (such as $\mathrm{Cu}$ ). When cycling performance is described in the manuscript, information on the current density (e.g., $\mathrm{mA} \mathrm{cm}^{-2}$ or $\mathrm{mA} \mathrm{cm}^{-3}$ ) and specific capacity for each cycle should be reported [22].

In the case of separators, various physicochemical characterization measurements should be reported, such as: evaluation of the MacMullin number, electrolyte uptake, thermal analysis, shut-down tests, shrinkage and tensile strength evaluation [23]. Electrochemical compatibility tests with electrodes, such as EIS or deposition/stripping tests in the case of Li metal anodes, are required [24,25].

Research works reporting battery models need to give all details on the chosen form of mass, energy and momentum conservation. This applies to control volumes of all sorts, all properly defined. Manuscripts aiming to present a good (predictive) physical-chemical model that describes the transport of charge, mass and energy, valid for parts of a cell or for the whole cell, need to refer to standard or well accepted setups/systems in the literature, to document progress. This applies to models of a part of the battery (e.g., one of electrochemical reactions) or models of the full battery. New choices of forms of constitutive equations and rate laws of chemical or physical processes need to be examined in comparison with literature data or experiments. All relevant approximations used must be reported. For instance, it is not always stated that the temperature at all positions and under all conditions is assumed to be constant. More generally, all modeling/simulation results must be compared with experimental data, either originating from the same contribution or from existing contributions in the literature. When the electrochemical performance of an electrode material is studied, the behavior of the counter-electrode and electrolyte must be defined. Simulations that investigate the effect of material properties, cell geometric variables, or operating conditions on the cell or battery performance should preferably refer to the performance of a well-accepted configuration, to measure progress in the field.

For papers dealing with lithium-sulfur (Li-S) batteries, in addition to what has been already pointed out in the 'Good Practice for the Experimental Section', the total electrode thickness, the total amount of electrolyte solution and the volume (or mass) of electrolyte per gram of elemental sulfur must be reported [26]. Authors must specify if particular current collectors (e.g., mesh or porous) for the sulfur electrode are used during the electrochemical testing. Also, if lithium polysulfide species are employed instead of solid elemental sulfur, authors must specify the polysulfide concentration as well as the volume used. Another important point is the definition of coulombic efficiency in a Li-S cell. The cycle test in Li-S cells usually starts with a discharge step (i.e., lithiation of the sulfur electrode), and often the coulombic efficiency is calculated as the ratio between the charge capacity and the previous discharge capacity. As the sulfur is the limiting reagent in the Li-S system, it is suggested to calculate and report the coulombic efficiency as the ratio of (discharge capacity):(charge capacity) for the same cycle [27].

For research works on Li-air batteries, the thickness and the carbon mass loading of the air electrode should be reported. The capacity of Liair batteries can be reported in terms of specific gravimetric capacity (e. g., $\mathrm{mAh} \mathrm{g}^{-1}$ carbon). However, the areal capacity (e.g, $\mathrm{mAh} \mathrm{cm}^{-2}$ ) should also be listed for evaluation in practical use. Similarly, the current applied in Li-air batteries is expected to be reported in current density format (e.g., $\mathrm{mA} \mathrm{cm}^{-3}$ ). If the authors claim an extremely high energy Liair cell in their manuscript, both the porosity and the carbon loading of the air electrode need to be disclosed to estimate the parasitic weight from electrolyte intake within pores. The thickness of the lithium metal electrode also needs to be reported. Authors must also specify if air, dry air, pure oxygen or dry oxygen have been used when testing the air electrode.

\section{General remarks}

The Elsevier website states the following under the Duties of Authors regarding Originality and Acknowledgement of Sources:

"The authors should ensure that they have written entirely original works, and if the authors have used the work and/or words of others, that this has been appropriately cited or quoted and permission has been obtained where necessary. Proper acknowledgement of the work of others must always be given. Authors should cite publications that have influenced the reported work and that give the work appropriate context within the larger scholarly record. Information obtained privately, as in conversation, correspondence, or discussion with third parties, must not be used or reported without explicit, written permission from the source. Plagiarism takes many forms, from 'passing off' another's paper as the author's own paper, to copying or paraphrasing substantial parts of another's paper (without attribution), to claiming results from research conducted by others. Plagiarism in all its forms constitutes unethical behaviour and is unacceptable."

For further details please see: https://www.elsevier.com/about/poli cies/publishing-ethics.

Highlights consist of a small number of bullet points that concisely capture the novel results of your research as well as any new methods that were used during the study.

The graphs and figures should exhibit a consistent and homogeneous 
presentation; color codes and font sizes should be maintained throughout to enable good legibility of the experimental data.

The use of emphatic statements such as "superior", "excellent", "outstanding" should be limited. The performance of tested materials/ components should be benchmarked versus state-of-the-art materials/ components and compared to the relevant literature.

\section{References}

[1] R. Raccichini, A. Varzi, V.S.K. Chakravadhanula, C. Kübel, A. Balducci, S. Passerini, Enhanced low-temperature lithium storage performance of multilayer graphene made through an improved ionic liquid-assisted synthesis, J. Power Sources 281 (2015) 318-325, https://doi.org/10.1016/j.jpowsour.2015.01.183.

[2] P. Novák, D. Goers, L. Hardwick, M. Holzapfel, W. Scheifele, J. Ufheil, A. Würsig Advanced in situ characterization methods applied to carbonaceous materials, J. Power Sources 146 (2005) 15-20, https://doi.org/10.1016/j. jpowsour.2005.03.129.

[3] X. He, R. Wang, M.C. Stan, E. Paillard, J. Wang, H. Frielinghaus, J. Li, In situ investigations on the structural and morphological changes of metal phosphides as anode materials in lithium-ion batteries, Adv. Mater. Interfaces 4 (2017), 1601047, https://doi.org/10.1002/admi.201601047.

[4] J. Rana, M. Stan, R. Kloepsch, J. Li, G. Schumacher, E. Welter, I. Zizak, J. Banhart, $\mathrm{M}$. Winter, Structural changes in Li2MnO3 cathode material for Li-ion batteries, Adv. Energy Mater. 4 (2014), 1300998, https://doi.org/10.1002/ aenm. 201300998.

[5] J. Li, R. Klöpsch, S. Nowak, M. Kunze, M. Winter, S. Passerini, Investigations on cellulose-based high voltage composite cathodes for lithium ion batteries, J. Powe Sources 196 (2011) 7687-7691, https://doi.org/10.1016/j.jpowsour.2011.04.030.

[6] J. Shi, N. Ehteshami, J. Ma, H. Zhang, H. Liu, X. Zhang, J. Li, E. Paillard, Improving the graphite/electrolyte interface in lithium-ion battery for fast charging and low temperature operation: fluorosulfonyl isocyanate as electrolyte additive, J. Power Sources 429 (2019) 67-74, https://doi.org/10.1016/j.jpowsour.2019.04.113.

[7] R. Raccichini, L. Furness, J.W. Dibden, J.R. Owen, N. García-Aráez, Impedance characterization of the transport properties of electrolytes contained within porous electrodes and separators useful for Li-S Batteries, J. Electrochem. Soc. 165 (2018) A2741-A2749, https://doi.org/10.1149/2.0631811jes.

[8] R. Raccichini, M. Amores, G. Hinds, Critical review of the use of reference electrodes in Li-ion batteries: a diagnostic perspective, Batteries 5 (2019) 12, https://doi.org/10.3390/batteries5010012.

[9] R. Nölle, K. Beltrop, F. Holtstiege, J. Kasnatscheew, T. Placke, M. Winter, A reality check and tutorial on electrochemical characterization of battery cell materials: how to choose the appropriate cell setup, Mater. Today (2019), https://doi.org/ 10.1016/j.mattod.2019.07.002.

[10] F. Holtstiege, P. Bärmann, R. Nölle, M. Winter, T. Placke, Pre-lithiation strategies for rechargeable energy storage technologies: concepts, promises and challenges, Batteries 4 (2018) 4, https://doi.org/10.3390/batteries4010004.

[11] T. Risthaus, D. Zhou, X. Cao, X. He, B. Qiu, J. Wang, L. Zhang, Z. Liu, E. Paillard, G. Schumacher, M. Winter, J. Li, A high-capacity P2 Na2/3Ni1/3Mn2/3O2 cathode material for sodium ion batteries with oxygen activity, J. Power Sources 395 (2018) 16-24, https://doi.org/10.1016/j.jpowsour.2018.05.026.

[12] J.W. Dibden, N. Meddings, J.R. Owen, N. García-Aráez, Quantitative galvanostatic intermittent titration technique for the analysis of a model system with applications in lithium-sulfur batteries, ChemElectroChem 5 (2018) 445-454, https://doi.org/10.1002/celc.201701004.
[13] X. He, J. Wang, R. Wang, B. Qiu, H. Frielinghaus, P. Niehoff, H. Liu, M.C. Stan, E. Paillard, M. Winter, J. Li, A 3D porous Li-rich cathode material with an: in situ modified surface for high performance lithium ion batteries with reduced voltage decay, J. Mater. Chem. A. 4 (2016) 7230-7237, https://doi.org/10.1039/ c6ta01448h.

[14] K. Oldiges, J. Michalowsky, M. Grünebaum, N. von Aspern, I. Cekic-Laskovic, J. Smiatek, M. Winter, G. Brunklaus, Tetrahydrothiophene 1-oxide as highly effective co-solvent for propylene carbonate-based electrolytes, J. Power Sources 437 (2019), 226881, https://doi.org/10.1016/j.jpowsour.2019.226881.

[15] J. Kasnatscheew, M. Börner, B. Streipert, P. Meister, R. Wagner, I. Cekic Laskovic, $\mathrm{M}$. Winter, Lithium ion battery cells under abusive discharge conditions: electrode potential development and interactions between positive and negative electrode, J. Power Sources 362 (2017) 278-282, https://doi.org/10.1016/j. jpowsour.2017.07.044.

[16] X. He, B. Yan, X. Zhang, Z. Liu, D. Bresser, J. Wang, R. Wang, X. Cao, Y. Su, H. Jia, C.P. Grey, H. Frielinghaus, D.G. Truhlar, M. Winter, J. Li, E. Paillard, Fluorine-free water-in-ionomer electrolytes for sustainable lithium-ion batteries, Nat. Commun. 9 (2018) 5320, https://doi.org/10.1038/s41467-018-07331-6.

[17] C. de la Torre-Gamarra, G.B. Appetecchi, U. Ulissi, A. Varzi, A. Varez, S. Passerini, Na3Si2Y0.16Zr1.84PO12-ionic liquid hybrid electrolytes: an approach for realizing solid-state sodium-ion batteries? J. Power Sources 383 (2018) 157-163, https:// doi.org/10.1016/j.jpowsour.2017.12.037.

[18] A. Gurlo, R. Riedel, In situ and operando spectroscopy for assessing mechanisms of gas sensing, Angew. Chem. Int. Ed. 46 (2007) 3826-3848, https://doi.org/ 10.1002/anie.200602597.

[19] S.M. Bak, Z. Shadike, R. Lin, X. Yu, X.Q. Yang, In situ/operando synchrotron-based X-ray techniques for lithium-ion battery research, NPG Asia Mater. 10 (2018) 563-580, https://doi.org/10.1038/s41427-018-0056-z.

[20] P. Kitz, M. Lacey, P. Novák, E. Berg, Operando EQCM-D with simultaneous in situ EIS : new insights into interphase formation in Li-ion batteries, Anal. Chem. 91 (2019) 2296-2303, https://doi.org/10.1021/acs.analchem.8b04924.

[21] Y.-C. Chien, A.S. Menon, W.R. Brant, D. Brandell, M.J. Lacey, Simultaneous monitoring of crystalline active materials and resistance evolution in lithium-sulfur batteries, J. Am. Chem. Soc. 142 (2020) 1449-1456, https://doi. org/10.1021/jacs.9b11500.

[22] S.S. Zhang, X. Fan, C. Wang, A tin-plated copper substrate for efficient cycling of lithium metal in an anode-free rechargeable lithium battery, Electrochim. Acta 258 (2017) 1201-1207, https://doi.org/10.1016/j.electacta.2017.11.175.

[23] S.S. Zhang, A review on the separators of liquid electrolyte Li-ion batteries, J. Power Sources 164 (2007) 351-364, https://doi.org/10.1016/j. jpowsour.2006.10.065.

[24] J. Landesfeind, J. Hattendorff, A. Ehrl, W.A. Wall, H.A. Gasteiger, Tortuosity determination of battery electrodes and separators by impedance spectroscopy, J. Electrochem. Soc. 163 (2016) A1373-A1387, https://doi.org/10.1149/ 2.1141607jes.

[25] M.F. Lagadec, R. Zahn, V. Wood, Characterization and performance evaluation of lithium ion battery separators, Nat. Energy 4 (2019) 16-25, https://doi.org/ 10.1038/s41560-018-0295-9.

[26] B.D. McCloskey, Attainable gravimetric and volumetric energy density of Li-S and $\mathrm{Li}$ ion battery cells with solid separator-protected Li metal anodes, J. Phys. Chem. Lett. 6 (2015) 4581-4588, https://doi.org/10.1021/acs.jpclett.5b01814.

[27] J.W. Park, K. Ueno, N. Tachikawa, K. Dokko, M. Watanabe, Ionic liquid electrolytes for lithium-sulfur batteries, J. Phys. Chem. C 117 (2013) 20531-20541, https:// doi.org/10.1021/jp408037e. 